\title{
Coulisses
}

Revue de théâtre

13 | Hiver 1996

Varia

\section{Dürrenmatt et le théâtre}

\author{
Laurence Dahan-Gaïda
}

\section{OpenEdition}

Journals

Édition électronique

URL : http://journals.openedition.org/coulisses/3961

DOI : 10.4000/coulisses.3961

ISSN : 2546-9460

\section{Éditeur}

Presses universitaires de Franche-Comté

\section{Édition imprimée}

Date de publication : 1 janvier 1996

Pagination : $12-20$

ISSN : 1150-594X

\section{Référence électronique}

Laurence Dahan-Gaïda, " Dürrenmatt et le théâtre », Coulisses [En ligne], 13 | Hiver 1996, mis en ligne le 15 mars 2019, consulté le 02 octobre 2020. URL : http://journals.openedition.org/coulisses/3961 ; DOI : https://doi.org/10.4000/coulisses.3961

Ce document a été généré automatiquement le 2 octobre 2020.

Coulisses 


\title{
Dürrenmatt et le théâtre
}

\author{
Laurence Dahan-Gaïda
}

1 Dix jours avant sa mort en 1990, Dürrenmatt ${ }^{1}$ évoquait les deux thèmes majeurs de son œuvre $^{2}$ : d'une part, sa vision du monde humain comme quelque chose d'imprévisible, d'absurde, de grotesque; de l'autre, sa foi dans le rationalisme des Lumières et dans la science. Les deux thèmes sont liés : Dürrenmatt a en effet toujours cherché à définir les potentiels et les limites de la raison dans un monde où l'action rationnelle planifiée lui semblait être devenue impossible.

2 Pour l'essentiel, Dürrenmatt fonde sa conception de la rationalité sur une équivalence entre raison, science et activité critique ${ }^{3}$. La science moderne est en effet pour lui un modèle, parce qu'elle a renoncé aux explications globales pour se contenter d'hypothèses provisoires et partielles, toujours susceptibles d'être critiquées, améliorées ou même abandonnées. Elle favorise ainsi une exploration élective du réel, fondée non plus sur des vérités absolues mais sur l'émulation entre points de vue concurrents. La science a donc appris à s'exercer dans la conscience de ses limites et de sa variabilité historique. Et c'est ce qui lui donne sa valeur exemplaire. Cette conscience réflexive et critique est en effet la condition indispensable aux progrès de la connaissance.

3 Mais elle est aussi pour Dürrenmatt un modèle dont le théâtre peut s'inspirer. En effet, elle assure la promotion d'une rationalité dialogique, fondée sur l'échange et la polémique, qui peut être utilisée au théâtre pour alimenter la tension dramatique. Car «le dramaturge a besoin d'une contradiction, d'un tremplin, de la possibilité d'un conflit $»^{4}$. Et la science, avec sa logique foncièrement hypothétique, peut renouveler le schéma général de l'action conflictuelle : celle-ci ne naît plus alors d'une lutte entre des personnages défendant des intérêts opposés, mais d'un affrontement entre hypothèses contradictoires, entre différents modèles de la réalité. Le potentiel dramatique et le potentiel cognitif sont ainsi mis au service d'une même exigence: favoriser une connaissance plurielle du réel et une approche dynamique de la vérité.

4 La science est donc loin d'être un simple thème ou un objet de représentation privilégié pour Dürrenmatt : elle est le support épistémologique de sa pensée et de son écriture 
dramatique en même temps qu'un instrument heuristique, permettant de représenter le réel en fonction de modèles concurrents :

Quand les mathématiciens et les physiciens interrogent le monde, ils se limitent toujours à un domaine limité. Ils proposent de plus en plus souvent des hypothèses de travail, qui peuvent être confirmées ou falsifiées par l'expérience. (...) Comme les physiciens, je crée des modèles de relations humaines possibles. Pour donner une idée de ce qu'est la lumière, le physicien est contraint d'utiliser tantôt le corpuscule, tantôt l'onde, et de recourir ainsi à différentes formules ; $\mathrm{j}$ 'ai moi aussi besoin de plusieurs modèles sur la scène. Ces modèles, ce sont mes " hypothèses ", ma « méthode » pour donner à voir certains phénomènes de la « Nature humaine » 5.

5 La conception que se fait Dürrenmatt de la science lui a été inspirée par sa lecture de Karl Popper. Fervent lecteur de ses textes dans les années 60-70, il y a trouvé une théorie qui lui permettait d'unifier en un tout cohérent son scepticisme inné et sa foi dans la rationalité des Lumières.

\section{Dürrenmatt et Popper}

6 Popper $^{6}$ considère les théories scientifiques comme des «fictions " relevant plus de l'intuition que de la nécessité logique. La science contient donc des éléments irrationnels et ne peut plus être considérée comme détentrice de vérités absolues. Ses théories doivent être plutôt conçues comme des hypothèses "falsifiables » : à savoir comme des énoncés provisoires, toujours susceptibles d'être infirmés par l'expérience empirique. Ainsi la vérification n'est plus pour Popper le critère de la vérité scientifique, c'est la falsification qui hérite de ce rôle. En d'autres termes, il n'existe plus pour la science de " théorie vraie », seulement des théories dont la fausseté n'a pas encore été établie. La falsification ne fournit pas seulement à la science un nouveau critère de vérité, elle sert aussi de critère de démarcation entre science et non-science. La falsification exige en effet d'une théorie qu'elle se prête aux procédures de vérification, c'est-à-dire qu'elle soit susceptible d'être infirmée ou confirmée par des faits d'expérience. Tous les discours qui se soustraient à ce critère ne sont pour Popper que des "pseudo-sciences », comme par exemple la psychanalyse.

7 Ce principe, présent dans de très nombreux textes de Dürrenmatt, sert de pierre de touche à la mise à l'épreuve des idées. Il fournit une justification théorique à sa méfiance instinctive envers les idéologies qui prétendent détenir le monopole de la vérité et tendent ainsi à se transformer en dogme. Dürrenmatt définit le dogme comme un mode de pensée abstrait qui, au lieu d'adapter la théorie aux faits empiriques, manipule ces derniers de manière à ce qu'ils viennent confirmer les présupposés théoriques de départ. La science, à l'inverse, a développé une logique interactive entre données de l'expérience et spéculation théorique et c'est dans le contrôle permanent exercé par les faits sur la théorie qu'elle puise sa légitimité. La vérité n'est plus pour elle une finalité, simplement une idée régulatrice, qui engage la pensée dans un processus continu de recherche, lequel exige une prudence épistémologique toujours aux aguets.

8 Cette activité permanente de contrôle et de remise en question, exercée de l'intérieur par la science, est pour Dürrenmatt le modèle dont la vie politique devrait s'inspirer. A l'ère de la bombe atomique, il est à ses yeux devenu urgent de renoncer à tous les absolus et à tous les idéaux figés, pour privilégier l'émulation entre différents points de 
vue rivaux. Comme les conjectures scientifiques, les institutions politiques devraient être considérées comme des réalités hypothétiques, toujours susceptibles d'être testées, critiquées et changées. Dürrenmatt part du même constat que Popper: l'homme est toujours en situation potentielle d'erreur et ce n'est qu'à travers un échange constant avec autrui, une critique rationnelle systématique, qu'il parviendra à progresser vers la liberté et la justice. Ici comme en science, l'évolution dépend d'une ouverture épistémologique fondée sur le débat et l'échange, sur la recherche commune d'un consensus par l'argumentation rationnelle.

Ces prises de position de Dürrenmatt ne sont pas sans évoquer les théories d'Habermas sur l'agir communicationnel.

\section{Le théâtre et l'agir communicationnel}

Fidèle aux principes de la rationalité discursive, Habermas ${ }^{7}$ donne pour fondement à la raison un modèle dialogique de communication orientée vers le consensus. Il imagine un scénario idéal de dialogue continu, de négociation perpétuelle entre des citoyens unis par leur désir commun d'atteindre un accord, par le biais d'une argumentation rationnelle. Son modèle de «l'agir communicationnel » exige que les participants coordonnent leur plan d'action de manière consensuelle. Dans cette logique, chaque accord doit être évalué en termes de reconnaissance intersubjective : autrement dit, la communication réussit là où les joueurs s'accordent sur les règles du jeu et créent ainsi un terrain commun pour légitimer et réévaluer périodiquement le contrat qui les lie.

Tout comme Popper, Habermas fait de la connaissance un produit du discours critique et conçoit la politique comme un processus de communication et de critique constant. La notion d'agir communicationnel suggère la nécessité d'un système politique non autoritaire qui encourage la critique, le débat et la polémique comme moyens de résister aux dangers du dogmatisme. Le succès de ce projet dépend de la vitalité de la sphère publique, cet espace idéalisé, distinct des institutions politiques, qui doit ménager des possibilités de communication entre citoyens libres et égaux. Il implique également l'existence d'un langage commun, d'un discours obéissant aux règles de la rationalité discursive, orienté vers la vérité, la justice et la sincérité.

Or le théâtre peut représenter un potentiel pour l'agir communicationnel. A sa manière, il peut en effet contribuer à vitaliser la sphère publique et jouer un rôle dans le projet d'émancipation de la rationalité discursive. La comédie en particulier est pour Dürrenmatt un outil indispensable à la prise de conscience et à l'activité critique. Art de moralistes, la comédie est un moyen de séduire le public, de le " piéger » pour l'amener à écouter des choses qui autrement seraient difficiles à entendre. A ses yeux, la tragédie n'est plus possible car elle présuppose un monde achevé, aux contours fermement établis. Or le monde dans lequel nous vivons est un monde mouvant, changeant, contradictoire, «sans autre ordre que celui imposé par la peur de la bombe atomique ». Dans ce monde où le sens tend irrésistiblement à devenir non-sens et se transforme parfois en folie meurtrière, c'est à la comédie qu'il incombe de prendre en charge le tragique de l'existence. Or le monde humain, on s'en rappelle, se caractérise pour Dürrenmatt pas son aspect grotesque : c'est justement ce mélange de grotesque et de tragique qui, recouvrant le hiatus entre forme et contenu, pourra favoriser l'effet de distanciation et la prise de conscience du spectateur. 
Ces principes sont mis à l'œuvre dans deux œuvres qui, précisément, traitent de l'arme nucléaire et de la responsabilité morale des scientifiques et des politiques. Dans Les Physiciens (1961) et Achterloo $^{8}$ (1983), les thèses de Dürrenmatt sur la rationalité scientifique fournissent un soubassement épistémologique à la pensée mais aussi les ressorts de l'action et de la construction dramatique.

\section{Les Physiciens}

L'action des Physiciens ${ }^{9}$ se développe autour des raisons qui ont amené trois physiciens à choisir l'asile de fous comme lieu de séjour. Le décor de l'asile est celui d'une élégante villa transformée en clinique privée par Mlle Mathilde von Zahnd, vieille fille bossue et célèbre doctoresse issue d'une puissante famille du pays. Les fous sont au nombre de trois, trois physiciens qui se font appeler Möbius, Newton et Einstein. Ils seraient des fous modèles si l'un d'eux, puis les deux autres à sa suite, n'avaient étranglé son infirmière. Une enquête est ouverte qui, après maints coups de théâtre, révèle l'identité réelle des trois patients.

Moebius, savant génial, détenteur d'un savoir capable de livrer l'humanité à la destruction, s'est réfugié à l'asile afin de garder secrètes ses découvertes. Mais Newton et Einstein, de leurs vrais noms, Beutler (alias Kilton) et Ernesti (alias Eisler), se révèlent être des agents secrets au service de deux puissances ennemies, chargés de convaincre Moebius de travailler pour leur gouvernement respectif. Finalement, c'est Moebius qui, par la force de ses arguments, finira par convaincre ses deux collègues de se ranger à son côté :

Ou bien nous restons dans un asile, ou alors c'est le monde entier qui se transforme en asile de fous. Ou nous nous effaçons de la mémoire des hommes, ou c'est l'humanité qui s'éteint. (p. 88)

Mais leur projet reste vœu pieux : même l'asile n'est pas un lieu sûr, il est devenu un piège qui se referme sur les physiciens, à la faveur d'un ultime coup de théâtre. Tombées aux mains de Mathilde von Zahnd, la directrice de l'asile, les découvertes de Möbius sont désormais la propriété d'une « aliéniste folle » qui, à la tête de son «trust universel », projette de se rendre maître de l'univers.

17 La construction dramatique des Physiciens repose entièrement sur la structure du paradoxe. Paradoxe dont la figure la plus suggestive est celle du ruban de Moebius : une surface à deux dimensions qui pourtant ne possède qu'une seule face. La science est à l'image de cette surface paradoxale, à la fois réversible et privée de revers. En effet, la science a contribué à améliorer nos conditions d'existence autant qu'à emprisonner l'humanité dans une logique de domination et de dégradation. De même le savant est une figure paradoxale : il a pour tâche de servir le savoir et le progrès mais il n'est que trop souvent que l'esclave d'un pouvoir irrationnel. C'est là que réside tout le problème, dans ce retournement potentiel de la raison en déraison, du savoir en pouvoir, du progrès en destruction. Or même lorsqu'elle se veut " raisonnable », la science n'est pas à l'abri de la folie qui la guette : ainsi, la solution choisie par Moebius, le retrait hors de la vie sociale, se révèle incapable de constituer une réponse aux problèmes posés par le danger nucléaire. Cinq ans avant la rédaction de sa pièce, Dürrenmatt évoquait déjà le problème :

Il est impossible de tenir secret ce qui est pensable. Tout processus de pensée est répétable. Le problème de l'énergie nucléaire - la bombe atomique n'est qu'un cas 
particulier - ne peut être résolu que sur le plan international. Par l'union des scientifiques. (...) Mais il est impossible de faire du devoir de rester bête un principe esthétique. La question est de savoir comment les physiciens doivent se comporter dans le monde d'aujourd'hui et pas seulement les physiciens ${ }^{10}$

C'est pour répondre à cette question que Dürrenmatt a ajouté en appendice à sa pièce une liste de vingt et une propositions, qui constituent à la fois un diagnostic et l'esquisse d'un modèle d'action, engageant non seulement les scientifiques mais l'ensemble de la communauté humaine. Car la physique est pour Dürrenmatt quelque chose de trop difficile pour les seuls physiciens. La communauté scientifique ne peut venir seule à bout des problèmes posés par l'arme nucléaire. A fortiori, l'individu isolé. Pour Dürrenmatt; en effet, l'héroïsme individuel n'a plus aujourd'hui aucun sens : même le plus grand des génies ne pourrait, par ses sacrifices, changer le monde ou le sauver de la catastrophe. Dürrenmatt prend ainsi le contrepied de Brecht qui, dans son Galileo Galiléi ${ }^{11}$, réaffirmait sa confiance dans la valeur exemplaire que peut prendre l'action individuelle. Brecht accordait à ses physiciens un pouvoir potentiellement illimité, à condition que leur savoir s'assortisse d'une conscience sociale et politique. Pour Dürrenmatt, l'exigence représentée par la figure de Galilée - mettre la science au service de l'humanité - ne peut plus être satisfaite. Même si les physiciens étaient conscients de leur responsabilité envers la société, il n'y aurait de rédemption ni individuelle ni collective. C'est ce qu'illustre la figure de Möbius : ayant choisi de rester méconnu pour sauver l'humanité, il est cependant impuissant à changer le cours des choses. Dans le meilleur des cas, la conscience politique permet aux scientifiques de s'auto-déterminer, pas d'influencer de manière significative les processus historiques.

La solution de Dürrenmatt se trouve dans le vingt et unième point : « Le contenu de la physique concerne les physiciens, ses effets l'ensemble des hommes. Ce qui est l'affaire de tous doit être résolu par tous. Toute tentative individuelle de régler ce qui concerne l'ensemble des hommes, est condamnée à l'échec». La solution proposée par Dürrenmatt réside dans un dialogue ininterrompu des scientifiques avec le reste de la communauté humaine, dans une éthique fondée sur la rationalité discursive et l'activité communicationnelle. S'ils veulent agir sur le cours de l'histoire, les scientifiques doivent s'engager dans le réseau de pratiques sociales et d'échanges intersubjectifs qui sont à la base de toute vie en collectivité. Dürrenmatt semble ici rejoindre les vues d'Habermas, qui impute le déclin de la sphère publique à la privatisation de la vie et à la tentative de donner des solutions individuelles aux problèmes de l'existence. Pour Dürrenmatt comme pour Habermas, l'activité scientifique doit être soumise à des contraintes éthiques et sociales, elle doit s'impliquer dans le débat public et la recherche commune d'un consensus. C'est seulement à ce prix que l'équivalence entre science et raison pourra être maintenue et que la relation entre pouvoir et savoir pourra évoluer dans un sens plus démocratique. 


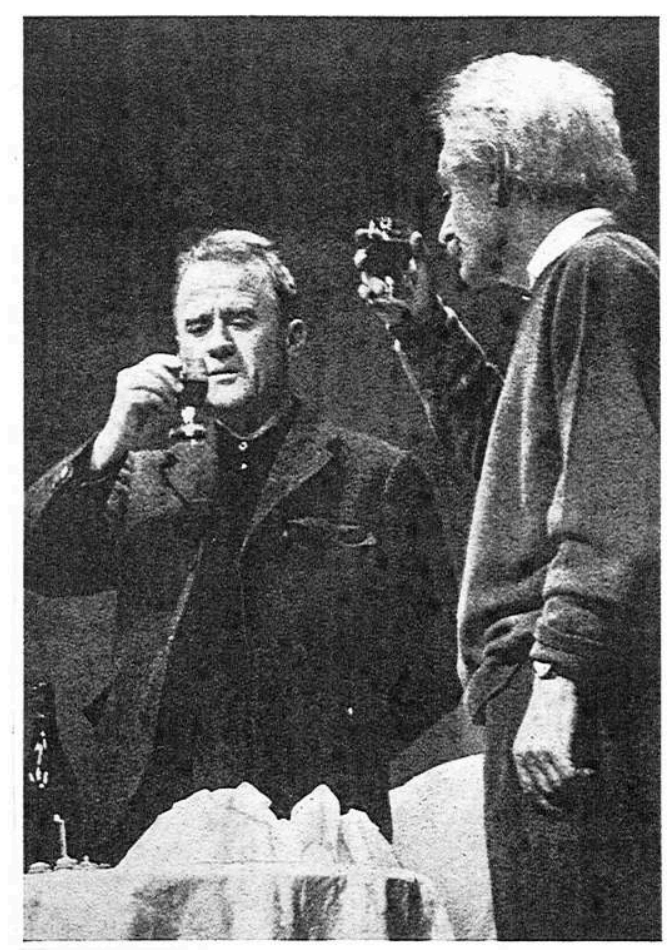

Royal Shakespeare Company, Londres 1963. Avec Cyril Cusack (Möbius) et Alan Webb (Einstein)

\section{Achterloo}

Réactualisant le schéma classique de la pièce dans la pièce, Achterloo reprend en l'inversant le schéma des physiciens. Möbius incarnait le seul être raisonnable dans un monde où la démence des gens " normaux » l'obligeait à se faire passer pour fou. Ici, ce sont les patients d'un asile de fous qui jouent sur scène le rôle de gens normaux, en l'occurrence de personnages historiques : Robespierre, Napoléon, Richelieu, etc. Le jeu de rôles, en mobilisant des figures notoires de tyrans ou d'utopistes, permet de révéler la «folie» de toutes les idéologies reposant sur des absolus. Autrement dit, la «folie » de toute action n'obéissant pas aux principes de la rationalité scientifique.

Dans l'œuvre de Dürrenmatt, les utopistes et les personnages en quête d'absolu sont toujours présentés comme des fous. Dürrenmatt exprime par là sa méfiance instinctive envers toutes les idéologies qui cherchent à restructurer l'ensemble de la société d'après des préceptes absolus. Comme Popper, il favorise la pensée inductive qui part des conditions de vie concrètes et non d'idéalités abstraites. Aux utopistes épris d'absolu, Popper oppose "l'ingénieur social», qui renonce aux schémas totalisants pour se contenter de réformes provisoires et "locales", toujours susceptibles d'être révisées ou améliorées. Foncièrement critique et hypothétique, cette attitude repose sur une logique de l'essai et de l'erreur et sur la mise en compétition de points de vue opposés, qui se critiquent et se relativisent mutuellement, empêchant ainsi toute fixation idéologique. 
L'action d'Achterloo est basée sur les événements qui ont mené à la loi martiale en Pologne en 1981. Dans la pièce jouée par les patients de l'asile, les absolutistes sont représentés par des versions modernes de Richelieu et de Robespierre. Promu au rang d'idéologue du Parti Communiste, Robespierre symbolise l'inadéquation des idéologies fondées sur des schémas abstraits, incapables de s'adapter aux conditions concrètes de l'existence. Ce penchant pour l'Absolu mène inéluctablement à l'oppression et au totalitarisme, comme en témoigne la figure de Richelieu. Pour mettre définitivement fin aux hérésies politiques et religieuses, ce dernier propose une coalition entre l'Eglise et le Parti. L'Union Marxiste-Catholique serait une solution universelle puisqu'elle réunirait les deux grands prétendants au monopole de la vérité et éliminerait ainsi toute possibilité d'opposition. Dans le second acte cependant, Richelieu est amené à une prise de conscience épistémologique qui lui fait reconnaître l'inhumanité de l'absolutisme. Et c'est dans la science qu'il va puiser le modèle de sa réflexion :

Un érudit quelconque, qui réfléchissait sur l'espace et le temps, écrivit un jour que dans la mesure où les principes mathématiques s'appliquent à la réalité, ils ne sont pas sûrs et que dans la mesure où ils sont sûrs, ils ne s'appliquent pas à la réalité. (p. 166)

L'érudit dont il est ici question est Albert Einstein: dans son cours Geometrie und Erfahrung $^{12}$, il soulignait la nature hypothétique de toute théorie en rappelant que la certitude était étrangère à la réalité concrète et vivante. Les conséquences s'imposent à Richelieu qui les transpose aux domaines de la théologie et de l'idéologie :

Ce que cet érudit inconnu a écrit vaut également pour la théologie et l'idéologie : dans le mesure où les principes théologiques et idéologiques s'appliquent à l'homme, ils ne sont pas sûrs, et dans la mesure où ils sont sûrs, ils ne s'appliquent pas à l'homme. Théologie et idéologie ne sont vraies que dans le vide. (p. 167)

Richelieu rejette ainsi toutes les idéologies reposant sur des absolus en faveur d'un réalisme politique, soucieux non pas de la «vérité» des idées, mais de leurs conséquences pratiques. Dans la pièce, c'est Napoléon qui tire les conclusions concrètes de cette conception : désireux d'éviter un affrontement nucléaire dont son pays serait la première victime, il choisit de trahir Jean Hus, le leader du syndicat indépendant, dont il partage pourtant les vues. Son soutien entraînerait en effet une invasion de son pays par l'Union soviétique, qui entraînerait à son tour une réaction de l'autre superpuissance et finirait par aboutir au conflit nucléaire.

25 Napoléon a donc choisi la raison, mais il finira assassiné par une jeune idéaliste, figure moderne de la Judith du mythe. Mais alors que Judith avait libéré son peuple en tuant Holopherne, Marion tue le seul être capable d'éviter à son pays la destruction. En subvertissant la signification de la figure mythique, Dürrenmatt montre une fois encore le caractère anachronique de l'héroïsme individuel et de toutes les formes d'idéalisme. Il n'existe pas pour lui de valeurs universelles, seulement des hypothèses qui doivent être mises à l'épreuve des changements historiques et des nouvelles réalités qu'ils créent. Et l'héroïsme individuel comme l'idéalisme sont de telles hypothèses. Ici encore, c'est la science qui sert de référence et de modèle : ouverte sur un espace de discussion et de critique ininterrompues, elle est un exemple moral pour la sphère politique et sociale.

Ainsi, malgré son scepticisme, Dürrenmatt parvient à « sauver » la raison en s'appuyant sur une épistémologie relativiste et hypothétique. Mais il tend par là à transformer la science en un discours "au-delà du bien et du mal », débarrassé de tout jugement de valeur et de tout présupposé idéologique. Pourtant la science elle-même a récusé le 
mythe d'un pur savoir, référé à un idéal de transparence et de désintéressement. Cette idéalisation de la science a rendu Dürrenmatt vulnérable aux attaques de scientisme dont il a parfois fait l'objet. Mais le modèle de rationalité qu'il défend reste cependant exemplaire par son ouverture à la critique et au dialogue : il reste fidèle à l'idéal d'une raison discursive capable d'articuler des significations authentiques dans une sphère publique irriguée d'échanges constants. Faisant siens les principes de communication, d'argumentation et de critique de la rationalité scientifique, son théâtre contribue à sa manière au dynamisme et à la pérennité du processus démocratique.

\section{BIBLIOGRAPHIE}

Les conceptions de Dürrenmatt sur la science et le théâtre s'expriment notamment dans les œuvres en prose et dans les essais suivants :

- Albert Einstein, éd. de l'Aire, Lausanne, 1982

- Sur Israël, Paris, Albin Michel, 1977

- La Mort de la Pythie, suivi de Minotaure, éd. de Fallois/L'Age d'Homme, 1990

- Ecrits sur le théâtre, Gallimard, 1970

- La mise en œuvres, Christian Bourgeois éd./Julliard/L'Age d'Homme, 1985

- Essais, 1988

Parmi ses principales œuvres dramatiques, on peut citer :

- Achterloo, précédé par Charlotte Kerr : Protocole d'une mise en scène fictive, Lausanne, éd. de L'Age d'Homme, 1989

- Les Physiciens, Lausanne, éd. de L'Age d'Homme, 1988

- La Visite de la vieille dame, Paris, Flammarion, 1956

- Romulus Le Grand, Lausanne, éd. de L'Age d'Homme, 1993

- Le Mariage de Mr Mississippi, Lausanne, éd. de l'Aire, 1973

- Le Météore, Lausanne, éd. de L'Age d'Homme, 1993

- Les Anabaptistes, Lausanne, éd. de L'Age d'Homme, 1993

\section{NOTES}

1. Friedrich Dürrenmatt, considéré comme l'un des dramaturges majeurs de notre temps, est né à Konolfingen, près de Bern en 1921. Fils de pasteur, il a fait des études de philosophie et de théologie avant de se consacrer à l'écriture et, plus particulièrement, à l'écriture théâtrale. Parallèlement, il s'est adonné avec passion à la peinture et au dessin. De 1952 jusqu'à sa mort en 1990 , il a vécu à Neuchâtel, où il a lui-même mis en scène une partie de ses pièces.

2. Dans une interview accordée à Michael Haller, parue dans Die Zeit, 28 décembre 1990. Cité par Joseph Federico, Confronting Modernity. Rationality, Science and Communication in German Literature of the 1980's, Camden House, Columbia, 1992, p. 15 («Face à la modernité : rationalité, science et communication dans la littérature allemande des années $80 »)$

3. Sur les rapports de Dürrenmatt avec la science, voir le livre déjà cité de Joseph Federico. Voir également l'article de Gerhard et Mona Knapp : «Reht - Gerechtigkeit - Politik. Zur Genese der 
Begriffe im Werk Friedrich Dürrenmatt » («Droit-Justice-Politique. La genèse des concepts dans l'œuvre de Friedrich Dürrenmatt»), dans Text + Kritik, 1977. Voir aussi de A.M. Wright, «Scientific Method and Rationality in Dürrenmatt " (« Méthode scientifique et rationalité chez Dürrenmatt ») dans German Letters n 35, 1981.

4. Paru dans Die Deutsche Woche du 18/1/1961. La traduction de l'allemand est la mienne, de même que dans tous les passages cités qui ne comportent pas d'indication de page.

5. Ibid.

6. Les thèses de Popper exposées ici se trouvent pour l'essentiel dans les ouvrages suivants : La Logique de la découverte scientifique (Payot, 1978), La Quête inachevée (Calmann-Lévy, 1981), Plaidoyer pour l'indéterminisme (Hermann, 1984)

7. Les thèses d'Habermas sur l'activité communicationnelle et sur la science se trouvent pour l'essentiel dans les ouvrages suivants: Morale et communication: conscience morale et activité communicationnelle (éd. du Cerf, 1986), La technique et la science comme idéologie (Gallimard, 1973), Théorie de l'agir communicationnel (Fayard, 1987)

8. II existe deux versions de cette pièce, l'une de 1983, l'autre de 1986. Cette dernière, comme le sous-titre l'indique (Ein Rollenspiel: un jeu de rôles), met l'emphase sur le jeu de rôles et sur ses implications psychologiques, esthétiques et théâtrales. C'est pourquoi nous commenterons ici la version de 1983, parue en français en 1989 à L'Age d'homme, précédée d'un texte de Charlotte Kerr : Protocole d'une mise en scène fictive.

9. Paru en allemand aux éditions Diogenes en 1962. Nous citons l'édition française parue à l'Age d'homme en 1988. La pièce a été montée en France en 1964 à la Comédie de l'Est.

10. Dans un article du 7 décembre 1956, paru dans Die Weltwoche, consacré au livre de l'écologiste Robert Jungk : Heller als tausend Sonnen («Plus clair que mille soleils »).

11. Pour une confrontation entre les deux pièces, voir l'article de Hans Mayer, « Dürrenmatt und Brecht oder die Zurùckname », (« Dürrenmatt et Brecht. Ou la rétractation »), dans Uber Friedrich Dürrenmatt, Zurich, Diogenes, 1980. Voir aussi l'article d'Ernst Schumacher, dans Theater der Zeit, 17, 1962, 5, pp. 68-71.

12. Albert Einstein, «Géométrie und Erfahrung ", Mein Weltbild, ("Géométrie et expérience ", dans « Ma vision du monde »), ed. Cari Selig, Berlin : Ullstein, 1989. La signification d'Einstein pour Dürrenmatt est centrale. Il lui a même consacré un livre, Albert Einstein, paru en français à Lausanne en 1982. 